\title{
Histomorphological and Morphometric Evaluation of Microvessel Density in Nodal Non-Hodgkin Lymphoma Using CD34 and CD105
}

\author{
Sucheta ${ }^{1}$ Sant Prakash Kataria ${ }^{1}$ Shivani Malik ${ }^{1} \quad$ Roomi Yadav $^{1} \quad$ Raman Kapil $^{1} \quad$ Rajeev Sen ${ }^{1}$ \\ ${ }^{1}$ Department of Pathology, Pt. B. D. Sharma Post Graduate Institute \\ of Medical Sciences, Rohtak, Haryana, India \\ Address for correspondence Sant Prakash Kataria, MBBS, MD, \\ Dr. Sant Prakash Kataria, Department of Pathology, \\ Pt. B. D. Sharma Post Graduate Institute of Medical Sciences, \\ Rohtak 124001, Haryana, India \\ J Lab Physicians 2021;13:22-28. \\ (e-mail: santpkataria@yahoo.com).
}

\begin{abstract}
Keywords

- angiogenesis

- CD105

- microvessel density

- non-Hodgkin

lymphoma
\end{abstract}

Background Expression of angiogenic markers determined by microvessel density (MVD) could be used as a reliable predictor of prognosis and as a potential target for antiangiogenic therapy in different categories of non-Hodgkin lymphoma (NHL).

Aims The aim of this study was to evaluate MVD using immunohistochemical methods and computer-assisted quantitative image analysis in nodal NHL patients and compare CD34 and CD105 expression in lymph nodes of NHL patients.

Materials and Methods The present study was conducted on 60 lymph node biopsies received in the Department of Pathology at our tertiary care center for histopathological examination. Representative paraffin-embedded tissue sections were stained with hematoxylin and eosin along with immunohistochemical stains for CD34 and CD105. MVDs were analyzed at 400× using automated image analyzer by two investigators independently.

Statistical Analysis Data were calculated, tabulated, and statistically analyzed using SPSS (Statistical Package for Social Studies) statistical program version 18. The values entered were mean of morphometric parameters. In all tests, $p$-values below 0.05 were regarded as significant.

Results MVD was determined by CD34 and CD105 antibody highly correlated with different categories of NHL. Higher MVD was observed in cases of aggressive NHL as compared with indolent $\mathrm{NHL}$ and the difference was statistically significantly. MVD using CD105 was correlated more strongly as compared to CD34 with different categories of NHL.

Conclusion The present study concluded that NHL exhibits potent angiogenic activity that increased significantly with increasing aggressiveness. The study also demonstrated that CD105 is more specific than CD34 as a marker of neoangiogenesis in NHL. published online May 22, 2021
DOI https://doi.org/

10.1055s-0041-1726569 ISSN 0974-2727. (c)2021. The Indian Association of Laboratory Physicians.

This is an open access article published by Thieme under the terms of the Creative Commons Attribution-NonDerivative-NonCommercial-License, permitting copying and reproduction so long as the original work is given appropriate credit. Contents may not be used for commercial purposes, or adapted, remixed, transformed or built upon. (https://creativecommons.org/licenses/by-nc-nd/4.0/).

Thieme Medical and Scientific Publishers Pvt. Ltd. A-12, 2nd Floor, Sector 2, Noida-201301 UP, India 


\section{Introduction}

Non-Hodgkin lymphoma (NHL) is defined by what it is not; that is, it includes all neoplasms of lymphoid origin other than Hodgkin lymphoma. NHL occurs five times more frequently than Hodgkin lymphoma. NHL occurs in both children and adults with an overall male:female ratio of 1.3:1. NHL usually presents as painless, localized, or generalized enlargement of lymph nodes with or without hepatosplenomegaly. Prognosis in NHL depends on many factors. An International Prognostic Index has been developed for aggressive NHL patients based on age, stage, number of extranodal sites of disease, performance status, and serum lactate dehydrogenase levels. Clinical factors such as sex, size of tumor, and $\beta$-2-microglobulin have been found to affect prognosis. Histologic grade also affects prognosis. In addition, a variety of factors may be of importance in determining prognosis, including proliferative rate, cytotoxic T cell response, loss of molecules of immune recognition, loss of cell adhesion antigens, gain of drug resistance molecules, acquisition of aneuploidy, gain of specific oncogenes, or loss of specific tumor suppressor genes and genomic imbalances. ${ }^{1}$ Extent of angiogenesis, measured as microvessel density (MVD), has also been studied as a prognostic factor. Several studies have shown that increased MVD is predictive of poor prognosis and is associated with high-grade tumor or high-grade transformation in NHLs. ${ }^{2}$ Other studies have failed to confirm these results. ${ }^{3}$

Angiogenesis, which is the development of new capillaries from existing blood vessels, occurs in both the developing embryo and postnatal life. ${ }^{4}$ The growth of solid tumors requires angiogenesis, leading to the development of microvessels (MVs); therefore, tumor expansion correlates with the extent of angiogenesis. ${ }^{5}$ MVs not only provide nutrients and oxygen but also remove catabolytic substances, while endothelial cells produce growth factors for tumor cells in a paracrine fashion. ${ }^{6}$ A well-developed microvascular system facilitates local expansion of tumor since endothelial cells secrete cellular matrix degrading enzymes. Angiogenic activity in a given tumor governs the potential for metastases and inhibition of angiogenesis may prove significant in suppressing neoplastic growth and invasiveness. ${ }^{7}$ Without adequate vascularization, tumors larger than $1 \mathrm{~mm}^{3}$ may undergo necrosis and cannot grow beyond a critical size or metastasize to another organ. Similarly, without an efficient blood supply, it is difficult to deliver anticancer drugs to all regions of a tumor in effective quantities. ${ }^{8}$ In tumors, the normal configuration of blood vessels is generally abolished. Large-caliber tumor vessels may have thin walls usually belonging to capillaries or an incomplete basement membrane and an unusual pericyte coat. The vessel wall is not always formed by a homogenous layer of endothelial cells. Instead, it may be lined with only cancer cells or a mosaic of cancer and endothelial cells.

The histological quantification of human tumor angiogenesis was introduced by Weidner et al in $1991 .{ }^{9} \mathrm{He}$ and his colleagues used immunohistochemical techniques to highlight tumor blood vessels with antibodies to Factor VIII-related antigen. Since then various other markers have been employed including CD31, CD34, and CD105. ${ }^{10}$ Highlighting the vessel wall made it possible to numerically count MVs, announcing the emerging of a new parameter that might be used in the characterization of solid tumors. Of the various methods to assess angiogenesis, MVD has been studied extensively in various tumors. ${ }^{11}$

Ever since, the quantification of tumor angiogenesis to predict tumor behavior has always been in the attention of pathologists. In this regard, the works of Folkman can be considered very important in establishing those parameters that are usually needed to be measured to describe angiogenesis. These parameters include vessel number, endothelial cell hyperplasia, and cytology, introducing for the first time the term intratumoral MVD. The reason why these parameters should be studied is to yield important information on the relationship to other clinicopathological tumor characteristics and help testing of antiangiogenic therapies. ${ }^{5}$

\section{Materials and Methods}

The present study was conducted on 60 lymph node biopsies performed due to various benign or malignant indications submitted in the Department of Pathology for histopathological examination. These 60 cases were divided into two groups. Group I or the control group included 20 cases of reactive lymphadenitis (RL). Group II included 40 cases of NHL that were divided according to the WHO classification and comprised of 21 cases of diffuse large B cell lymphoma (DLBCL, not otherwise specified [NOS]) along with six cases each of peripheral T cell lymphoma (PTCL, NOS), chronic lymphocytic leukemia/small lymphocytic lymphoma (CLL/SLL), mantle cell lymphoma (MCL), and one case of follicular lymphoma (FL). Representative paraffin-embedded tissue sections of all the cases were stained with routine hematoxylin and eosin along with immunohistochemical stains with monoclonal antibodies to CD34 at 1:50 dilution (Dako) and CD105 at 1:25 dilution (Dako). "Hotspots," that is, areas of maximal MVD, were identified. In four such hotspots, vessel number and MVD were analyzed at 400× using automated image analyzer by two investigators independently. The mean value of both investigators was considered as the MVD in each case. Sections of lymph node were used as positive controls for anti-CD34 and anti-CD105 antibodies. Negative controls were prepared by replacing the antibody by phosphate-buffered saline or nonimmune serum.

The quantitative morphometric studies were done by image analysis. Images provided by a charged device video camera coupled with Olympus BX51 microscope at a magnification $400 \times$ were stored on a host computer based on Pentium 4 processor with operating system Microsoft Windows Vista/XP through a digital frame grabber and processing was done by image analysis software Image Pro Plus Version 6.3.

MV was defined as any highlighted endothelial cell or endothelial cell cluster clearly separate from adjacent MVs, tumor cells, and other connective tissue elements. Vessel lumens were not necessary for a structure to be defined as MV. MVD was assessed by light microscopy in representative areas of sections with highest numbers of capillaries and small venules (neovascular "hotspots") according to the 
method that was first described by Weidner et al. ${ }^{9}$ The microsections stained with CD34 and CD105 were scanned first at low magnification $(100 \times)$, and after the most intense areas of neovascularization (hotspot) were identified in sections, MV counts were done on a minimum of four fields at $400 \times$. Computer-assisted image analysis was performed on all cells, tissues, and vessels expressing antibody staining, avoiding confounding background, and including all positive staining vessels, whereas contaminating areas were excluded using the specific computer function. Data was collected as the number of MVs in 400× field. Mean of four microscopic fields was taken and MVD per $\mathrm{mm}^{2}$ was calculated. Finally, all the data was tabulated in Excel sheet from which mean and median were calculated in all categories of NHLs.

Data were calculated, tabulated, and statistically analyzed using SPSS (Statistical Package for Social Studies) statistical program version 18 . The values entered were mean of morphometric parameters. In all the tests, $p$-values below 0.05 were regarded as significant. For comparison between immunohistochemical staining between CD34 and CD105, chi-squared test was applied. To compare the number of MVs and MVD between RL and various categories of NHL, $t$-test was applied.

\section{Results}

A total of 60 cases of lymph node biopsies were included in the present study that were divided into groups. Group I included 20 (33.33\%) cases of RL and Group II included $40(66.67 \%)$ cases of NHL. Maximum cases of RL ( $n=8,40 \%)$ were in the age group of 30 to 40 years followed by age group of 20 to 30 years ( $n=4,20 \%$ ). The mean age for RL was 30 years. However, maximum cases of $\mathrm{NHL}(n=10,25 \%)$ were in the age group of 60 to 70 years followed by the age group of 50 to 60 years ( $n=8,20 \%$ ). Most common presentation in both the groups was cervical lymphadenopathy followed by inguinal lymphadenopathy in cases of RL and axillary lymphadenopathy in cases of NHL. No significant difference was observed in the MVDs from lymph nodes at different sites. Among NHL majority cases $(n=21,52.5 \%)$ were of DLBCL, NOS type followed by six (15\%) cases each of PTCL, NOS, CLL/SLL, and MCL type and one (2.5\%) case of FL. Thus, there were 27 cases of aggressive lymphomas (DLBCL and PTCL) and 13 cases of indolent lymphomas (CLL/SLL, MCL and FL).

The total number of vessels evaluated in the study were 5,657 with CD34 and 1,173 with CD105. While using CD34, the total number of vessels counted were 3,019 in cases of RL, 1,517 in DLBCL, 567 in PTCL, 189 in CLL/SLL, 311 in MCL, and 54 in FL. On using CD105 as the endothelial marker, the number of vessels counted were 227 in cases of RL, 515 in DLBCL, 182 in PTCL, 99 in CLL/SLL, 128 in MCL, and 22 in FL. Although we had only one case of FL in our study, a difference was observed in the MVD between follicular and interfollicular areas of reactive lymph nodes as well as in FLs and MVD was more in the interfollicular areas in both cases.

Mean MVDs calculated using CD34 and CD105 in various categories of NHL are shown in - Table $\mathbf{1}$ and the difference between mean MVDs in various categories came out to be statistically significant.
Table 1 Mean MVDs at 400× using CD34 and CD105 antibodies in various categories of non-Hodgkin lymphoma

\begin{tabular}{|l|l|l|}
\hline \multirow{2}{*}{ Type of NHL } & \multicolumn{2}{|l|}{ Mean MVD $\left(\mathrm{no} . / \mathrm{mm}^{2}\right)$} \\
\cline { 2 - 3 } & CD34 & CD105 \\
\hline DLBCL, NOS & $75.25 \pm 6.89$ & $25.55 \pm 3.62$ \\
\hline PTCL, NOS & $98.44 \pm 8.09$ & $31.60 \pm 3.59$ \\
\hline CLL/SLL & $32.82 \pm 7.24$ & $17.19 \pm 3.88$ \\
\hline MCL & $53.99 \pm 9.85$ & $22.22 \pm 3.27$ \\
\hline
\end{tabular}

Abbreviations: CLL/SLL, chronic lymphocytic leukemia/small lymphocytic lymphoma; DLBCL, NOS, diffuse large B cell lymphoma, not otherwise specified; MVD, microvessel density; NHL, non-Hodgkin Lymphoma; PTCL, NOS, peripheral T cell lymphoma, not otherwise specified.

The mean MVDs using CD34 and CD105 were highest in PTCL (most aggressive NHL) followed by DLBCL and MCL. However, low MVD was observed in CLL/SLL (indolent NHL) patients. This observation shows that MVDs correlate well with the aggressiveness of NHL.

The mean MVDs in case of RL and NHL (all categories combined) at 400× using CD34 and CD105 are shown in (-Fig. 1). In cases of RL, majority of the MVs were identified in paracortical areas as compared with germinal centers. In cases of RL ( $\boldsymbol{- F i g s . ~} 2$ and $\mathbf{3}$ ), the difference between MVD evaluated using CD34 and CD105 came out to be significant ( $p=0.003$ ). On comparing mean MVDs of Group I (RL) and Group II (NHL) cases (-Fig. 2) using CD34, increased MVD of 157.5 \pm 7.49 per $\mathrm{mm}^{2}$ was observed in cases of RL. However, in case of NHL, the mean MVD with CD34 was $68.70 \pm 28.13$ per $\mathrm{mm}^{2}$ ( - Fig. 4). This difference of MVDs in the two groups was statistically significant $(p=0.01)$. On the contrary, mean MVD evaluated using CD105 was comparatively much less in cases of RL, that is, $11.67 \pm 4.70$ per $\mathrm{mm}^{2}$. On using CD105 in cases of NHL, the mean MVD was $24.64 \pm 3.6$ per mm² $^{2}$ (-Fig. 5). This difference in the two groups was also statistically significant $(p=0.04)$. This shows that CD105 is more specific for tumor-related angiogenesis as compared with CD34.

\section{Discussion}

The NHLs are a diverse group of lymphoproliferative neoplasms with variable clinical behavior and prognosis. Currently, lymphomas are classified on the basis of morphology, immunology, and genetic and clinical features. The clinical stage of the lymphoma is the most important prognostic factor, apart from the histological grade (indolent versus aggressive). ${ }^{11}$ Highlighting the vessel wall using various endothelial markers made it possible to numerically count MVs, announcing the emerging of a new parameter that might be used in the characterization of hematolymphoid malignancies and other solid tumors. Although immunohistochemical staining for pan endothelial markers clearly highlights the vascular endothelium, endoglin (CD105) is suggested to be a useful marker for evaluating the angiogenesis. Of the various methods to assess angiogenesis, MVD has been studied extensively in various tumors. ${ }^{9}$ There is paucity of literature regarding comparison of neoangiogenesis markers in NHL and RL. The reason why these parameters should be studied 


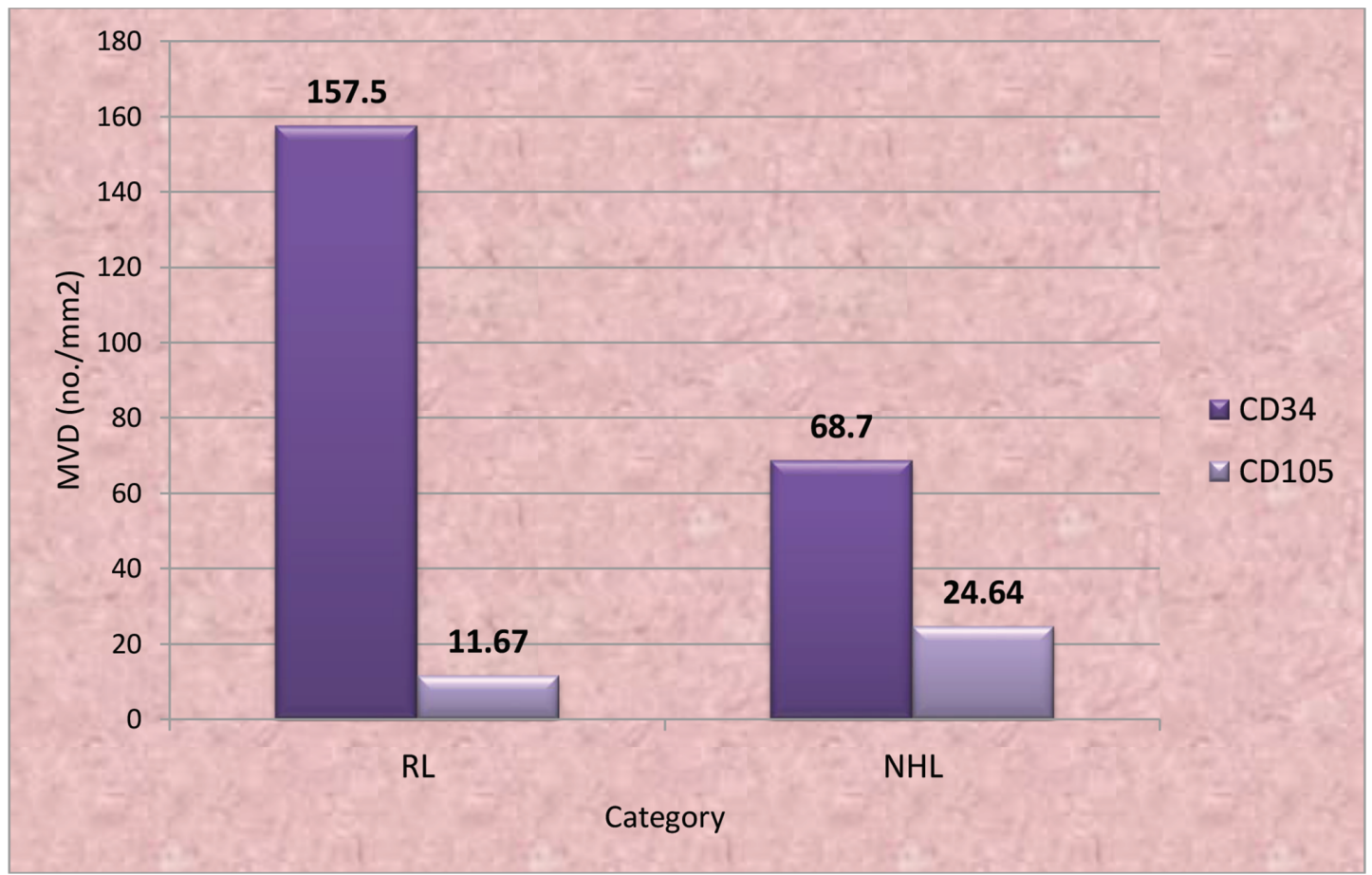

Fig. 1 Comparison of mean MVDs using CD34 and CD105 antibodies in Group I (RL) and Group II (NHL). MVD, microvessel density; NHL, non-Hodgkin lymphoma; RL, reactive lymphadenitis.

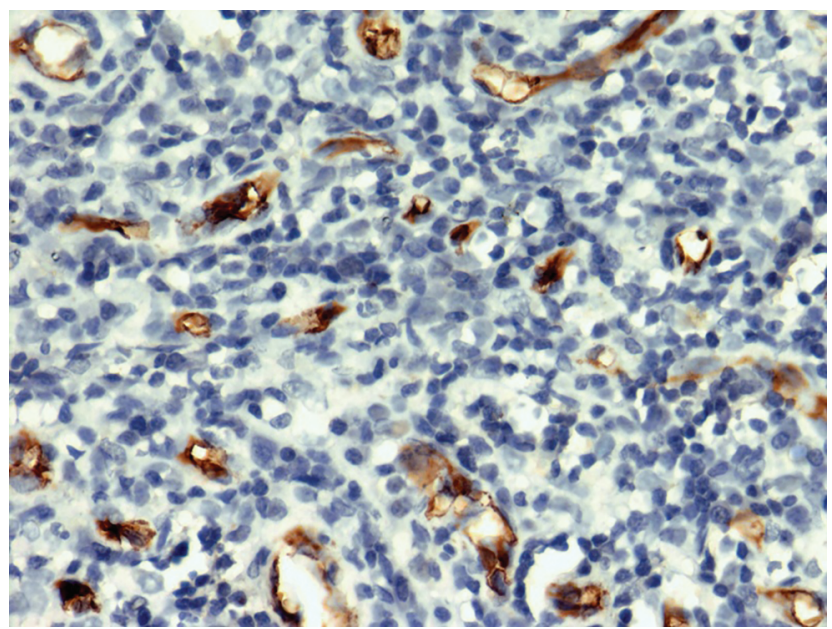

Fig. 2 Microvessels in reactive lymphadenitis (CD34; 400×).

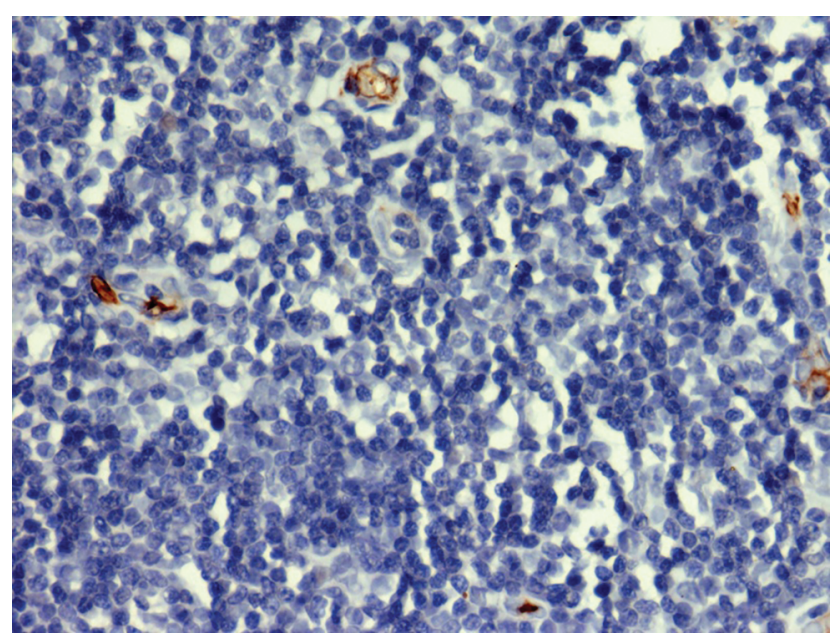

Fig. 3 Microvessels in reactive lymphadenitis (CD105; 400×).

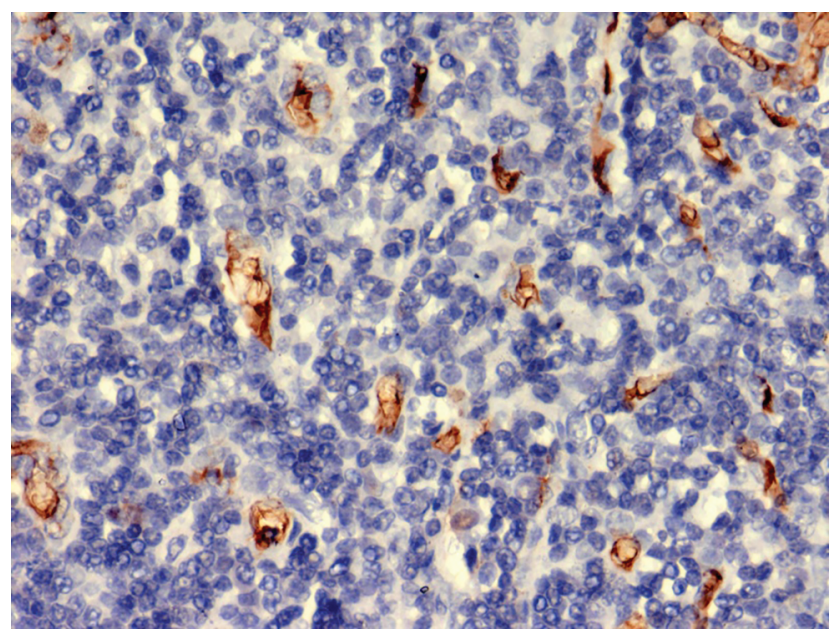

Fig. 4 Microvessels in peripheral T cell lymphoma (CD34; 400×).

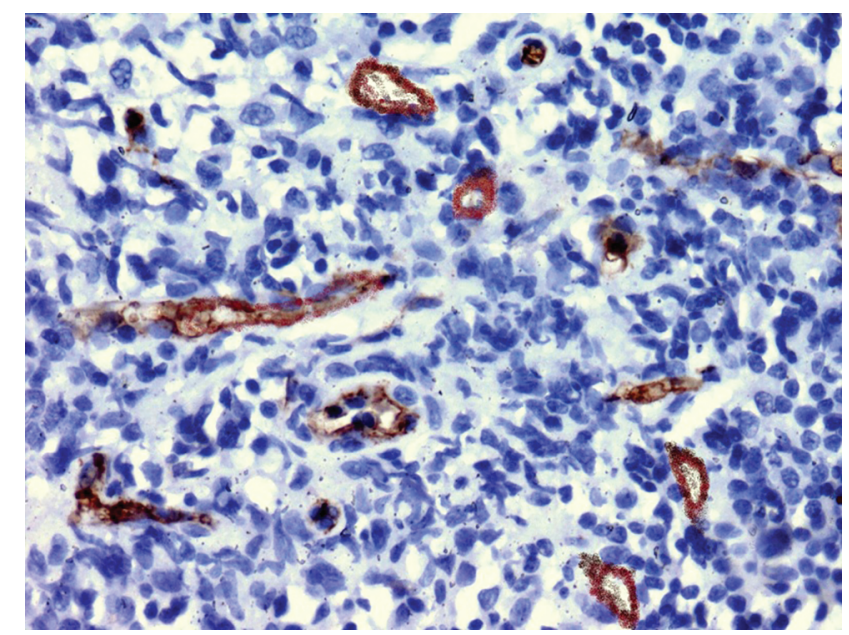

Fig. 5 Microvessels in peripheral T cell lymphoma (CD105; 400×). 
is to yield important information on the relationship to other clinicopathological tumor characteristics and help testing of antiangiogenic therapies. ${ }^{5}$

\section{Comparison of Microvessel Density in Reactive Lymphadenitis}

Increased MVD was observed using CD34 in cases of RL, which was comparable to studies performed by Aggarwal et $\mathrm{al}^{11}$ and Ruan et $\mathrm{al}^{12}$ who revealed the similar pattern of increased MVDs in RL ( - Table 2).

A comparison of angiogenetic activity using CD34 and CD105 was attempted in our study. There were only few studies in which CD105 was used as an angiogenesis marker in RLs as it was supposed to be a marker of neoangiogenesis in tumors. A low MVD of $11.67 \pm 4.70$ per $\mathrm{mm}^{2}$ using CD105 was observed in RL as compared with NHL. Study performed by Duse et $\mathrm{al}^{13}$ analyzed the results expressing only a percentage of vessels staining positive for CD105 and observed decreased percentage (12.82\%) of positive vessels in RL. Our study was in concordance with the study performed by above-mentioned author revealing similar findings.

\section{Comparison of Microvessel Density in Various Categories of Non-Hodgkin Lymphoma}

Angiogenesis plays a key role in tumor growth, invasiveness, and progression. Few studies have been performed in NHL for the demonstration of neoangiogenesis. Ribatti et $\mathrm{al}^{14}$ reported increased capillary proliferation in lymph node biopsies of high-grade NHL. MVD has been shown to correlate with

Table 2 Mean MVDs in RL

\begin{tabular}{|l|l|l|}
\hline & $\begin{array}{l}\text { Endothelial } \\
\text { marker }\end{array}$ & MVD \\
\hline \multirow{2}{*}{ Our study } & CD34 & $156.25 \pm 7.49 / \mathrm{mm}^{2}$ \\
\cline { 2 - 3 } & CD105 & $11.67 \pm 4.70 / \mathrm{mm}^{2}$ \\
\hline Aggarwal et al11 & CD34 & $191.92 \pm 12.16 / \mathrm{mm}^{2}$ \\
\hline Ruan et al ${ }^{12}$ & CD34 & $47.92 \pm 13.05$ per $200 \times$ \\
\hline
\end{tabular}

Abbreviations: MVD, microvessel density; RL, reactive lymphadenitis. biological behavior in nodal B cell NHL and the frequency of NHL tissue MVs increased with pathological progression.

Ridell and Norrby showed increased MVD in involved lymph nodes of patients with SLL but the number of blood vessels did not correlate with the grade of tumor. ${ }^{15}$

Arias and Soares found a statistically significant difference in MVD measured by immunostaining with Factor VIII-related antigen between low- and high-grade NHL when classified in either the working formulation or the Kiel classification. ${ }^{16}$

Another study performed by Ribatti et al in 1996 observed highest MVD in aggressive type of lymphomas including Burkitt's lymphoma and PTCL compared with intermediate and DLBCL and lower in indolent FL. ${ }^{17}$

Data on angiogenesis in B cell lymphomas is controversial partially owing to the application of different lymphoma classification systems. As B cell lymphomas encompass heterogenous diseases, angiogenic events are likely to differ in various entities. ${ }^{18}$

We observed increased MVD using CD34 as a marker of angiogenesis in aggressive lymphomas as compared with indolent lymphomas and this difference between the MVDs was statistically significant.

Cases of DLBCLs showed increased MVD of $75.25 \pm 6.89$ per $\mathrm{mm}^{2}$ using CD34. Similar findings were observed by Agarwal et $\mathrm{al}^{11}$ and Tzankov et $\mathrm{al}^{19}$ in patients of DLBCL.

PTCL is an aggressive type of NHL showing increased angiogenesis. Literature reviewed showed only one study demonstrating angiogenesis in PTCL using CD34. We have also attempted to calculate the MVD in PTCL to know the pattern of angiogenesis. Highest MVD (98.44 $\left.\pm 8.09 / \mathrm{mm}^{2}\right)$ using CD34 was observed that was comparable to the study performed by Aggarwal et al ${ }^{11}$ who also revealed an increased MVD in cases of PTCL using CD34 (- Table 3).

Indolent lymphomas are slowly progressive tumors showing a low angiogenetic activity. Our study also showed decreased MVD of $32.82 \pm 7.24$ per $\mathrm{mm}^{2}$ using CD34 in CLL/SLL that was comparable to the studies by Aggarwal et al ${ }^{11}$ and Tzankov et al $^{19}$ who also showed low MVD in indolent lymphomas that was in concordance with our study.

Table 3 Mean MVDs in various categories of NHL

\begin{tabular}{|c|c|c|c|c|c|}
\hline & \multirow[t]{2}{*}{ Endothelial marker } & \multicolumn{4}{|l|}{ MVD } \\
\hline & & DLBCL, NOS & PTCL, NOS & CLL/SLL & $\mathrm{MCL}$ \\
\hline \multirow{2}{*}{$\begin{array}{l}\text { Our study } \\
\text { (per } \mathbf{m m}^{2)}\end{array}$} & CD34 & $75.25 \pm 6.89$ & $98.44 \pm 8.09$ & $32.82 \pm 7.24$ & $53.99 \pm 9.85$ \\
\hline & CD105 & $25.55 \pm 3.62$ & $31.60 \pm 3.59$ & $17.19 \pm 3.88$ & $22.22 \pm 3.27$ \\
\hline Aggarwal et al11 & CD34 & $149.91 \pm 13.68$ & $183.42 \pm 8.24$ & $76.78 \pm 10.41$ & - \\
\hline Tzankov et al ${ }^{19}$ & CD34 & $31 \pm 17$ & - & $19 \pm 12$ & $19 \pm 7$ \\
\hline $\begin{array}{l}\text { Ruan et al }{ }^{12} \\
\text { (per 200×) }\end{array}$ & CD34 & $37.10 \pm 16.24$ & - & $59.18 \pm 17.30$ & - \\
\hline \multirow[t]{2}{*}{ Mazur et $a^{20}$} & CD34 & Aggressive NHL & \multicolumn{3}{|c|}{$19.45 \pm 11.24$} \\
\hline & & Indolent NHL & \multicolumn{3}{|c|}{$21.7 \pm 12.4$} \\
\hline
\end{tabular}

Abbreviations: CLL/SLL, chronic lymphocytic leukemia/small lymphocytic lymphoma; DLBCL, NOS, diffuse large B cell lymphoma, not otherwise specified; MCL, mantle cell lymphoma; MVD, microvessel density; NHL, non-Hodgkin lymphoma; PTCL, NOS, peripheral T cell lymphoma, not otherwise specified. 
Similarly, lower MVD (53.99 $\left.\pm 9.85 / \mathrm{mm}^{2}\right)$ was observed in cases of MCL that was also in concordance with the study conducted by Tzankov et $\mathrm{al}^{19}$ ( $\mathbf{- T a b l e} \mathbf{3}$ ).

However, Mazur et $\mathrm{al}^{20}$ found no significant correlation between MVD revealed by CD34 staining and the grade of histological malignancy in the lymph nodes of patients with NHL based on the Revised European American Lymphoma (REAL) classification. Ruan et $\mathrm{al}^{12}$ also showed a reversed pattern of MVD in CLL/SLL by expression of neoangiogenetic markers using CD34/Smooth Muscle Actin (SMA) as compared with RL. This may be due to perivascular and intrastromal areas that were stained with CD34 as well as SMA as there is increase in stromal cells in indolent subtype of CLL/SLL.

On using CD105 as the angiogenesis marker, the highest MVD was seen in cases of PTCLs $\left(31.60 \pm 3.59 / \mathrm{mm}^{2}\right)$ followed by DLBCLs $\left(25.55 \pm 3.62 / \mathrm{mm}^{2}\right), \operatorname{MCL}\left(22.22 \pm 3.27 / \mathrm{mm}^{2}\right)$, and $\mathrm{CLL} / \mathrm{SLL}\left(17.19 \pm 3.88 / \mathrm{mm}^{2}\right)$. This difference in MVDs was also statistically significant.

Duse et $\mathrm{al}^{13}$ reported a high heterogeneity of blood vessels in NHLs for the first time. They used CD105/SMA and CD34/SMA double immunostaining for highlighting tumor blood vessels for the categorization of immature and intermediate type of blood vessels and observed that use of CD105 for counting of blood vessels in lymphomas is more sensitive and can appreciate in a more accurate fashion the angiogenic status of NHL as compared with other markers.

Various authors ${ }^{21-24}$ performed MV counts using different angiogenic markers in various tumors and revealed that MVD increased with varying degrees of differentiation and grades of tumors.

Our findings were in concordance with the studies conducted by Weidner et al, ${ }^{21}$ Netto et al, ${ }^{22}$ Nayha et al, ${ }^{23}$ and Laitakari et al ${ }^{24}$ showing an increase in MVD in high-grade NHLs.

\section{Comparison of CD34 and CD105 as a Marker of Angiogenesis}

Present study revealed that CD105 appeared to be more specific and sensitive markers for various tumor angiogenesis. Our study was supported by authors ${ }^{25-29}$ who used CD105 antibody for calculating MVD in breast carcinoma, hepatocellular carcinoma, small cell lung cancer, nervous system lymphomas, and colorectal adenoma-carcinoma sequence as compared with CD34 antibody.

In the present study, as we did not have any information regarding patients' follow-up, we could not determine the relationship between MVD and survival. But our study showed that there was statistically significant difference in total MVD between different categories of NHL with a pattern of increasing angiogenesis in aggressive lymphomas. Thus, it was suggested that MVD could be used as a measure of angiogenic activity and aggressiveness in different categories of NHL.

If the prognostic value of quantitative assessment of tumor angiogenesis is confirmed in a large prospective study in addition to conventional pathologic assessment, MVD could be a practical and valuable standard prognostic test in predicting patient outcome in patients with NHL and in selecting patients for systemic adjuvant therapy, especially in the use of future antiangiogenic drugs.

The MVD using CD105 antibody was lower than that of MVD-CD34 in respective grades. This is due to the fact that CD34 is a nonspecific marker and stains all MVs including some perivascular stromal cells and lymphatic vessels, which may have contributed to falsely elevated MVD values. In contrast, CD105 stains small, newly formed tumor vessels only. Thus, CD105 appears to be more specific and sensitive marker for tumor angiogenesis. The study also demonstrated the superiority of CD105 over CD34 as a marker of neoangiogenesis in NHL, which is consistent with studies of breast cancer, lung cancer, and hepatocellular carcinoma. ${ }^{27,28,30}$

\section{Conclusion}

The present study concluded that NHLs exhibit potent angiogenic activity that increased significantly with increasing aggressiveness. MVD determined by CD34 and CD105 antibody was highly correlated with different categories of NHL. Higher MVD was observed in cases of aggressive NHL as compared with indolent NHL. These findings further suggested the potential significance of characteristics of MVD as potential prognostic marker as well as its application in improved selection of patients for antiangiogenic and other treatments. In the present era of newer antiangiogenic therapies, NHLs' angiogenesis and alteration in vascular morphology definitely have prognostic significance and thus help to improve the treatment modalities and patient care.

\section{Financial Support and Sponsorship \\ None.}

\section{Contribution of Authors}

Sucheta was involved in designing, definition of intellectual content, literature search, data acquisition, data analysis, and manuscript preparation.

Sant Prakash Kataria did conceptualization, data analysis, manuscript editing, and manuscript review.

Shivani Malik was involved in statistical analysis.

Roomi Yadav did literature search.

Raman Kapil did data analysis and literature search.

Rajeev Sen did conceptualization and editing.

\section{Note}

The manuscript has been read and approved by all the authors and the requirements for authorship have been met. Each author believes that the manuscript represents honest work.

\section{Conflicts of Interest}

The authors have no conflicts of interest to declare.

\section{References}

1 Chang KL, Arber DA, Weiss LM, Lymph nodes and spleen. In: DeLellis RA, Frable WT, Li Volsi VA, Wick MR, eds. Silverberg's Principles and Practice of Surgical Pathology and Cytopathology. 5th edition. Vol. 1, Cambridge (UK): Cambridge University Press; 2015: 688-812 
2 Bellamy WT, Richter L, Frutiger Y, Grogan TM. Expression of vascular endothelial growth factor and its receptors in hematopoietic malignancies. Cancer Res 1999;59(3):728-733

3 Hazar B, Paydas S, Zorludemir S, Sahin B, Tuncer I. Prognostic significance of microvessel density and vascular endothelial growth factor (VEGF) expression in non-Hodgkin's lymphoma. Leuk Lymphoma 2003;44(12):2089-2093

4 Asahara T, Murohara T, Sullivan A, et al. Isolation of putative progenitor endothelial cells for angiogenesis. Science 1997;275(5302):964-967

5 Folkman J. Tumor angiogenesis. Adv Cancer Res 1985;43: 175-203

6 Hamada J, Cavanaugh PG, Miki K, Nicolson GL. A paracrine migration-stimulating factor for metastatic tumor cells secreted by mouse hepatic sinusoidal endothelial cells: identification as complement component C3b. Cancer Res 1993;53(18):4418-4423

7 Benjamin LE, Golijanin D, Itin A, Pode D, Keshet E. Selective ablation of immature blood vessels in established human tumors follows vascular endothelial growth factor withdrawal. J Clin Invest 1999;103(2):159-165

8 Carmeliet P, Jain RK. Angiogenesis in cancer and other diseases. Nature 2000;407(6801):249-257

9 Weidner N, Folkman J, Pozza F, et al. Tumor angiogenesis: a new significant and independent prognostic indicator in early-stage breast carcinoma. J Natl Cancer Inst 1992;84(24):1875-1887

10 Mezei T, Horváth E, Turcu M, Gurzu S, Raica M, Jung I. Microvascular density in non-Hodgkin B-cell lymphomas measured using digital morphometry. Rom J Morphol Embryol 2012;53(1):67-71

11 Aggarwal D, Srivastava G, Gupta R, Pant L, Krishan G, Singh S, Angiogenesis in Non-Hodgkin's lymphoma: an intercategory comparison of microvessel density. ISRN Haematol; 2012: 943089

12 Ruan J, Hyjek E, Kermani P, et al. Magnitude of stromal hemangiogenesis correlates with histologic subtype of non-Hodgkin's lymphoma. Clin Cancer Res 2006;12(19):5622-5631

13 Duşe AO, Ceauşu RA, Cîmpean AM, Ioniță H, Raica M, Gaje P. Heterogeneity of malignant non-Hodgkin lymphoma-associated blood vessels. Rom J Morphol Embryol 2012;53(4):985-988

14 Ribatti D, Vacca A, Marzullo A, et al. Angiogenesis and mast cell density with tryptase activity increase simultaneously with pathological progression in B-cell non-Hodgkin's lymphomas. Int J Cancer 2000;85(2):171-175

15 Ridell B, Norrby K. Intratumoral microvascular density in malignant lymphomas of B-cell origin. APMIS 2001;109(1):66-72

16 Arias V, Soares FA. Vascular density (tumor angiogenesis) in non-Hodgkin's lymphomas and florid follicular hyperplasia: a morphometric study. Leuk Lymphoma 2000;40(1-2):157-166
17 Ribatti D, Vacca A, Nico B, Fanelli M, Roncali L, Dammacco F. Angiogenesis spectrum in the stroma of B-cell non-Hodgkin's lymphomas. An immunohistochemical and ultrastructural study. Eur J Haematol 1996;56(1-2):45-53

18 Koster A, Raemaekers JM. Angiogenesis in malignant lymphoma. Curr Opin Oncol 2005;17(6):611-616

19 Tzankov A, Heiss S, Ebner S, et al. Angiogenesis in nodal B cell lymphomas: a high throughput study. J Clin Pathol 2007;60(5):476-482

20 Mazur G, Wróbel T, Dziegiel P, Jeleń M, Kuliczkowski K, Zabel M. Angiogenesis measured by expression of CD34 antigen in lymph nodes of patients with non-Hodgkin's lymphoma. Folia Histochem Cytobiol 2004;42(4):241-243

21 Weidner N, Carroll PR, Flax J, Blumenfeld W, Folkman J. Tumor angiogenesis correlates with metastasis in invasive prostate carcinoma. Am J Pathol 1993;143(2):401-409

22 Netto GC, Bleil CB, Hilbig A, Coutinho LM. Immunohistochemical evaluation of the microvascular density through the expression of TGF- $B(C D$ 105/endoglin) and CD 34 receptors and expression of the vascular endothelial growth factor (VEGF) in oligodendrogliomas. Neuropathology 2008;28(1):17-23

23 Näyhä V, Viitanen T, Stenbäck F. Altered extent, pattern and characteristics of microvascular density are indicators of neoplastic progression in the endometrium. Int J Cancer 2005;115(6):975-980

24 Laitakari J, Näyhä V, Stenbäck F. Size, shape, structure, and direction of angiogenesis in laryngeal tumour development. J Clin Pathol 2004;57(4):394-401

25 Kumar S, Ghellal A, Li C, et al. Breast carcinoma: vascular density determined using CD105 antibody correlates with tumor prognosis. Cancer Res 1999;59(4):856-861

26 Yao Y, Pan Y, Chen J, Sun X, Qiu Y, Ding Y. Endoglin (CD105) expression in angiogenesis of primary hepatocellular carcinomas: analysis using tissue microarrays and comparisons with CD34 and VEGF. Ann Clin Lab Sci 2007;37(1):39-48

27 Tanaka F, Otake Y, Yanagihara K, et al. Evaluation of angiogenesis in non-small cell lung cancer: comparison between anti-CD34 antibody and anti-CD105 antibody. Clin Cancer Res 2001;7(11):3410-3415

28 Sugita Y, Takase Y, Mori D, Tokunaga O, Nakashima A, Shigemori M. Endoglin (CD 105) is expressed on endothelial cells in the primary central nervous system lymphomas and correlates with survival. J Neurooncol 2007;82(3):249-256

29 Akagi K, Ikeda Y, Sumiyoshi Y, et al. Estimation of angiogenesis with anti-CD105 immunostaining in the process of colorectal cancer development. Surgery 2002;131(1, Suppl):S109-S113

30 Beresford MJ, Harris AL, Ah-See M, Daley F, Padhani AR, Makris A. The relationship of the neo-angiogenic marker, endoglin, with response to neoadjuvant chemotherapy in breast cancer. Br J Cancer 2006;95(12):1683-1688 